\title{
OFDM: From the Idea to Implementation
}

\author{
S. A. Fechtel \\ Infineon Technologies AG, Rosenheimer Str. 116, 81699 München, Germany
}

\begin{abstract}
OFDM (orthogonal frequency-division multiplexing) is one of the key digital communication technologies of the current decade. The first part of this paper presents the fundamentals of OFDM and its benefits in the presence of multipath propagation in a tutorial-like fashion. The second part details on some of the most important aspects of OFDM transceiver implementation: concept of receiver channel filtering and $\mathrm{A} / \mathrm{D}$ conversion, radio impairment compensation (I/Q mismatch), and OFDM demodulator (FFT) design.
\end{abstract}

\section{Introduction}

The ever-increasing demand for very high-rate wireless data transmission calls for technologies which make use of the available electromagnetic resource in the most intelligent way. Key objectives are spectrum efficiency (bits per second per Hertz), robustness against multipath propagation, range, power consumption, and implementation complexity. These objectives are often conflicting, so techniques and implementations are sought which offer the best possible tradeoff between them.

Two of the most effective means of closing the gap between the achieved performance and channel capacity are advanced channel coding (to combat noise) and OFDM modulation (to combat multipath). In conjunction with advanced forward error correction (FEC) coding, e.g., turbo, spherical, or LDPC (low-density parity check) codes, advanced OFDM is the modulation of choice when it comes to improving robustness against multipath - possibly fading - at reasonable cost of implementation. In order to improve spectral efficiency, the best strategy is to increase the capacity of the channel itself. This is effectuated by multiple-input multipleoutput (MIMO) smart antenna systems which make use of the spatial dimension to essentially scale the data rate transferred over the same channel bandwidth with the number of antennas. As MIMO systems have now become within reach of low-cost implementation in highly-integrated devices, they are currently a very active field of R\&D activity and standardization.

Correspondence to: S. A. Fechtel

(stefan.fechtel@infineon.com)
Combining several advanced technologies leads to particularly attractive solutions. OFDM, which by now has matured to an established technology, also serves as a solid basis of many of the most recent R\&D developments, in particular MIMO. Figure 1 gives an overview of some important wireless communication standards and standardization efforts in terms of range and PHY (physical layer) data rate. OFDM and MIMO-OFDM are seen to play a key role in high-rate data transmission over wireless channels for a wide range of applications such as WLAN (wireless local-area networks), DVB-T (terrestrial digital video broadcasting), and also UWB (ultra wideband).

Concentrating on wireless LAN in this paper, Table 1 displays basic parameters of current and forthcoming IEEE WLAN standards. All standards but the very first $(802.11 \mathrm{~b}$, $1 \ldots 11 \mathrm{Mb} / \mathrm{s}$ ) are based on OFDM. Currently, 802.11a/gbased devices are entering the mass market, offering PHY rates between 6 and $54 \mathrm{Mb} / \mathrm{s}$. In the near future, 802.11n standardization will be finalized. The two most promising proposals are TGn Sync (task group n synchronization) and WWiSE (world-wide spectrum efficiency). TGn Sync proposes doubling the channel bandwidth to $40 \mathrm{MHz}$ (rates up to $630 \mathrm{Mb} / \mathrm{s}$ ), whereas WWiSE confines itself to $20 \mathrm{MHz}$ channels $(40 \mathrm{MHz}$ optional where permitted, then with rates up to $540 \mathrm{Mb} / \mathrm{s}$ ) in order to obey current spectrum regulations. Both contending proposals improve MAC efficiency, and, more importantly, both are based on MIMO-OFDM technology with between $2 \times 2$ and $4 \times 4$ TX and RX antennas. The latest development efforts for next-generation WLAN, e.g., the WIGWAM (wireless Gigabit with advanced multimedia support) project initiated in 2003, are targeting wideband MIMO-OFDM (100 MHz channel in higher bands up to $60 \mathrm{GHz}$ ) to attain scalable data rates well beyond $1 \mathrm{~Gb} / \mathrm{s}$.

This paper is organized as follows: Sect. 2 features a brief tutorial on OFDM and its benefits in the presence of multipath radio propagation, and Sect. 3 gives details on some of the most important aspects of a typical OFDM-based WLAN PHY transceiver implementation, viz., concept of receiver channel filtering and subsequent $\mathrm{A} / \mathrm{D}$ conversion, transmitter I/Q mismatch calibration as an example of radio impairment compensation, and the design of OFDM modulators and demodulators, i.e., FFT design. 


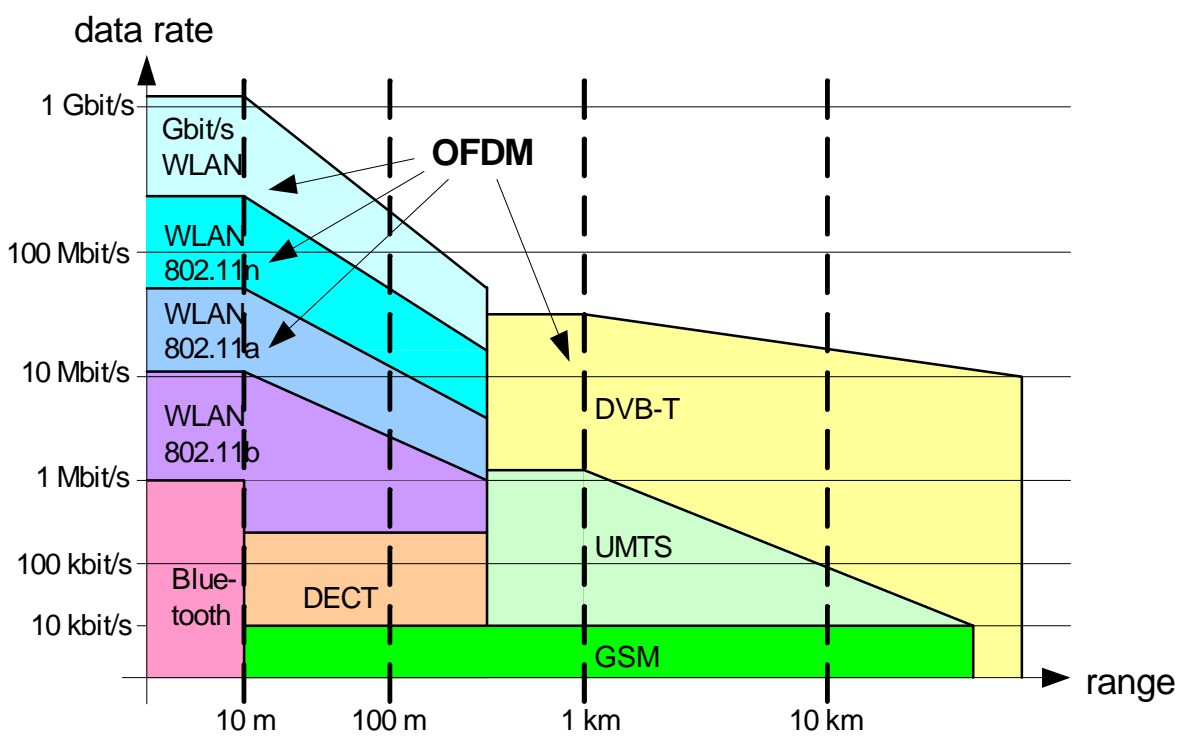

Fig. 1. Wireless Communication Standards.

Table 1. Basic Parameters of Wireless LAN Standards and Standard Proposals.

\begin{tabular}{lll}
\hline Standard & Band, Channel, Modulation & maximum PHY rates \\
\hline $802.11 \mathrm{~b}$ & $2.4 \mathrm{GHz}, 25 \mathrm{MHz}$ channel, DSSS/CCK & $11 \mathrm{Mb} / \mathrm{s}$ \\
$802.11 \mathrm{a} / \mathrm{g}$ & $5 / 2.4 \mathrm{GHz}, 20 \mathrm{MHz}$ channel, OFDM & $54 \mathrm{Mb} / \mathrm{s}$ \\
$802.11 \mathrm{n}$ & $5 / 2.4 \mathrm{GHz}, 20 / 40 \mathrm{MHz}$ channel, OFDM & \\
TGn Sync & $20 \mathrm{MHz}$ (optional), 40 MHz (mandatory) & $140 / 315 \mathrm{Mb} / \mathrm{s}(2 \times 2,20 / 40 \mathrm{MHz})$ \\
Proposal & $2 \times 2$ (mandatory) or 4x4 MIMO OFDM & $280 / 630 \mathrm{Mb} / \mathrm{s}(4 \times 4,20 / 40 \mathrm{MHz})$ \\
WWiSE & $20 \mathrm{MHz}$ (mandatory), $40 \mathrm{MHz}$ (optional) & $135 / 270 \mathrm{Mb} / \mathrm{s}(2 \times 2,20 / 40 \mathrm{MHz})$ \\
Proposal & $2 \times 2$ (mandatory) or 4x4 MIMO OFDM & $270 / 540 \mathrm{Mb} / \mathrm{s}(4 \times 4,20 / 40 \mathrm{MHz})$ \\
Gbit/s & $5 / 17 / 24 / 60 \mathrm{GHz}$ bands, $100 \mathrm{MHz}$ channel, & more than $1 \mathrm{~Gb} / \mathrm{s}$ \\
WLAN & up to 4x4 MIMO OFDM & \\
\hline
\end{tabular}

\section{Fundamentals of OFDM}

Wireless communication usually deals with multipath radio channels. The time- and frequency domain representations of a typical channel realization are shown in Fig. 2. The baseband-equivalent channel impulse response $h(\tau ; t)$ and transfer function $H(f ; t)$ both characterize the instantaneous channel state at time $t$, where the impulse response is formed by superposition of a (possibly large) number of reflected or scattered multipath rays with distinct (complex-valued) gains $c_{i}(t)$ and delays $\tau_{i}(t)$. Naturally, the gains tend to become smaller as the path delay rises, and the set of "relevant" paths with gain $c_{i}(t)$ above a certain significance level (depending on the required signal-to-noise ratio for the particular link) determines the effective span $\tau_{\max }$ of the multipath channel.

Wideband transmission channels used in high-rate communications are often strongly frequency-selective (lower part of Fig. 2), rendering conventional equalization difficult, both in terms of performance and complexity. An old but effective idea to circumvent time-domain equalization consists in converting a bitstream with high rate $R$ into a large number of $N$ parallel bitstreams, each with low rate $R / N$, and transmitting the set of $N$ substreams over a corresponding set of $N$ smallband subchannels formed by partitioning the broadband channel, see Fig. 3. The receiver essentially performs $N$ parallel demodulations, one for each frequency bin, followed by parallel-to-serial conversion to restore the original serial high-rate bitstream. This frequency partitioning approach is called frequency-division multiplex (FDM). In order to avoid crosstalk between subchannels, their carrier waveforms are usually chosen to be mutually orthogonal. The modulation so formed is termed orthogonal FDM, or OFDM. Computationally efficient IFFT (inverse FFT) and FFT (fast Fourier transform) architectures are usually implemented to perform OFDM modulation and demodulation, respectively. 

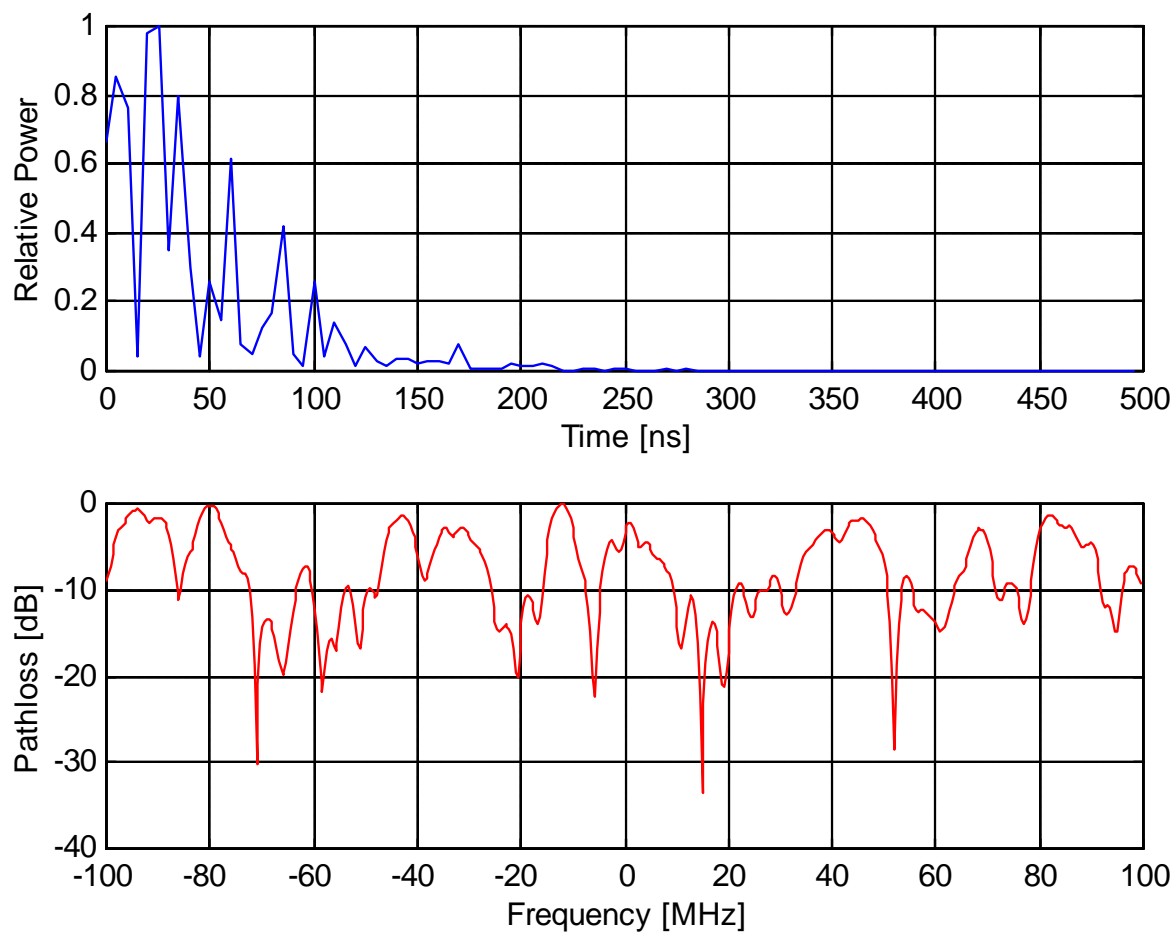

Fig. 2. Typical Multipath Radio Channel Impulse Response and Transfer Function.

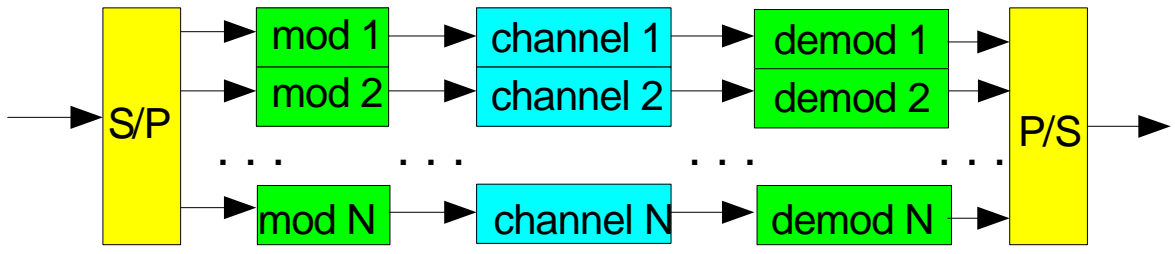

Fig. 3. Parallel Transmission over Smallband Subchannels.

Figure 4 illustrates OFDM transmission over a frequencyselective channel. In this simple example, the four subcarrier waveforms are given by $0,1,2$, and 3 full periods of sinusoids within length $T$, respectively. An OFDM symbol is formed by modulating each of these subcarriers by a (complex-valued) PSK or QAM symbol and superpose the resulting modulated waveforms. The corresponding frequency-domain signals (right-hand side of Fig. 4) exhibit a $\sin (x) / x$-characteristic with center frequencies $0,1 / T, 2 / T$ and $3 / T$, respectively. At these frequencies, all but one waveform are seen to have zero crossings, so they are mutually orthogonal. Given a sufficiently large number of subchannels, thus small subcarrier bandwidth relative to the channel coherence bandwidth, the use of OFDM effectively converts a frequency selective channel into an orthogonal set of frequency-flat subchannels.

Beyond orthogonality, a second ingredient is required in making OFDM symbols robust against multipath, viz., the guard interval. Figure 5 illustrates the insertion of a guard interval and the effect of multipath on a so-formed OFDM symbol. The guard interval of length $T_{g}$ is given by the cyclic extension at the beginning of an OFDM symbol (left-hand side of Fig. 5), its samples are taken from the latter section of the (original) OFDM symbol. Hence, the total OFDM symbol length increases to $T+T_{g}$. The associated loss in transmission efficiency $T /\left(T+T_{g}\right)$ is greatly outweighted by the benefits in the presence of multipath, as illustrated by the right-hand side of Fig. 5: at the channel output (here tworay), delayed and weighted copies of the waveform (the one with frequency $1 / T$ is shown as an example) are superimposed. Therefore, the received waveforms exhibit discontinuities at the (delayed) borders between consecutive OFDM symbols. However, as long as the span of channel delays $\tau_{\max }$ is smaller than guard interval length $T_{g}$, all of these discontinuities fall inside the guard interval. By simply eliminating the guard interval samples and retaining only the "useful" signal, the received waveforms turn out to be given by 


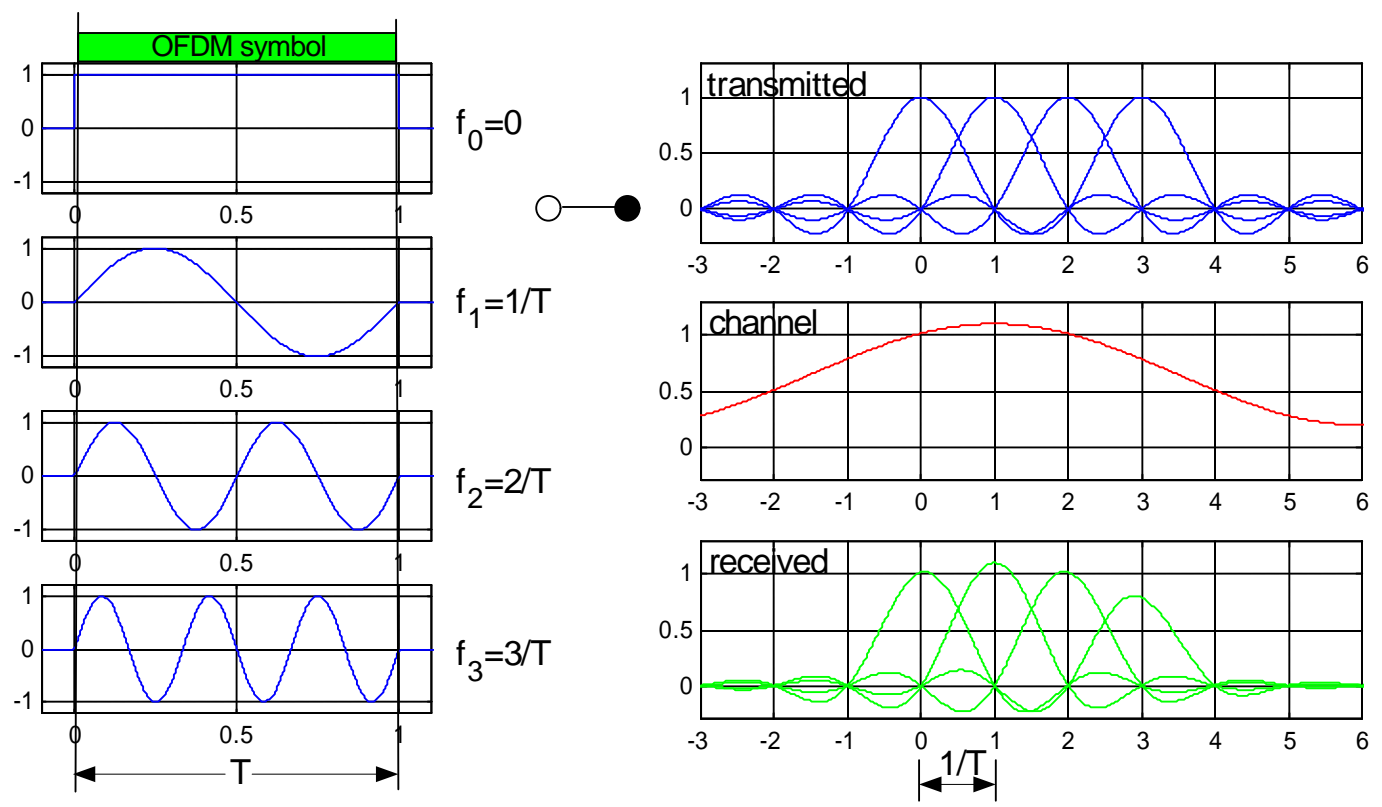

Fig. 4. OFDM Symbol: Orthogonal Subcarrier Waveforms.
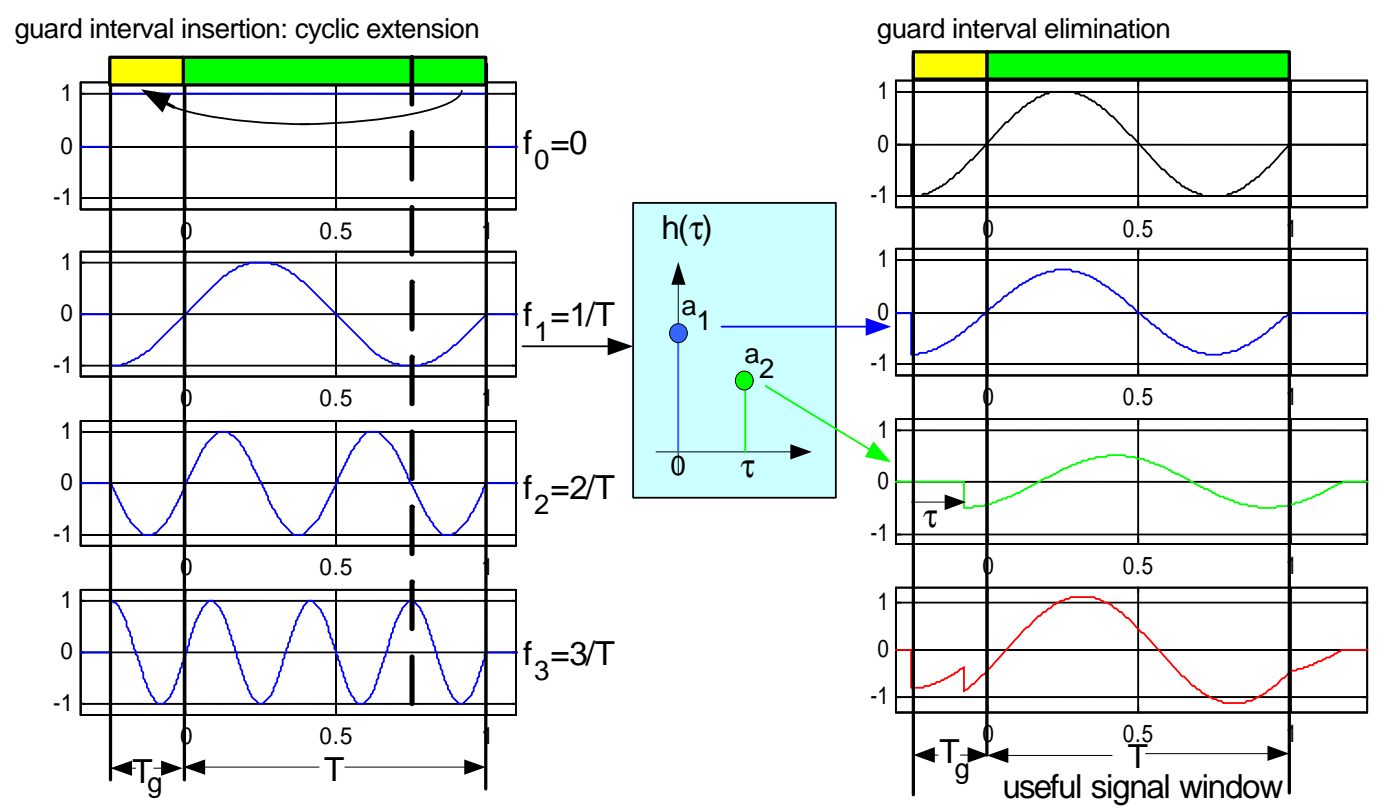

Fig. 5. OFDM Symbol: Guard Interval.

the amplitude-weighted and phase-shifted versions of their original counterparts. The orthogonality property of the original OFDM symbol is thus restored, which implies both orthogonality in time (no intersymbol interference, ISI) and in frequency (no intercarrier interference, ICI). As a consequence, equalization in the subcarrier domain becomes particularly simple: the remaining amplitude and phase distortion (or, equivalently, the complex-valued gain factor) affecting each subcarrier can be easily corrected for, e.g., by complex division or demapping.

\section{OFDM Transceiver Implementation}

In this second part of the paper, we focus our attention to WLAN transceiver implementation. Figure 6 shows the block diagram of a $2.4 / 5.2 \mathrm{GHz}$ dual-band $802.11 \mathrm{a} / \mathrm{b} / \mathrm{g}$ transceiver which has been realized as a three-chip solution. The transceiver comprises (from left to right) the host interface, MAC (medium access controller), PHY with A/D, $\mathrm{D} / \mathrm{A}$ converters and OFDM/CCK baseband processors, RF transceiver, power amplifier, and two diversity antennas with 


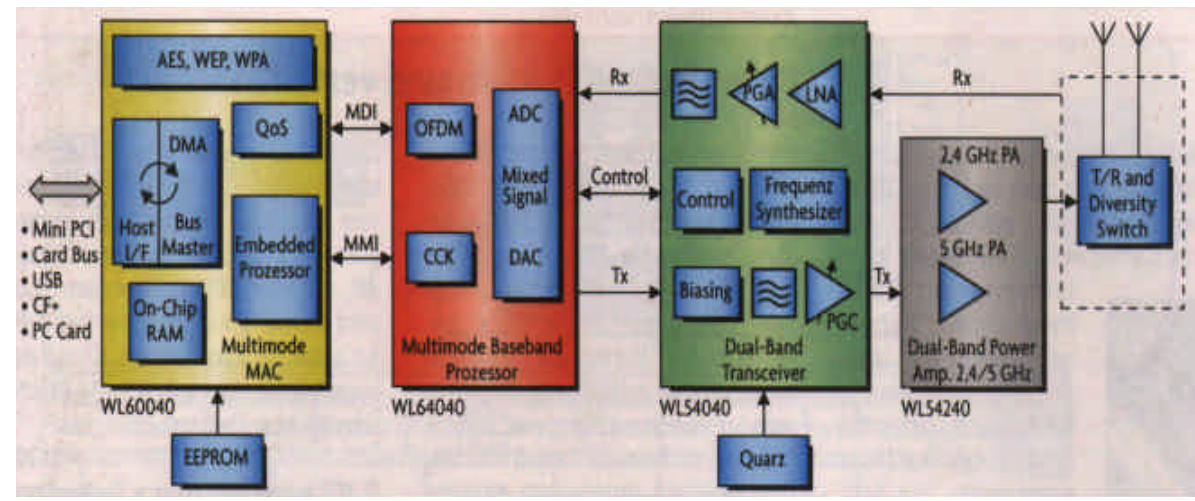

Fig. 6. $802.11 \mathrm{a} / \mathrm{b} / \mathrm{g}$ Dual-Band WLAN Transceiver.
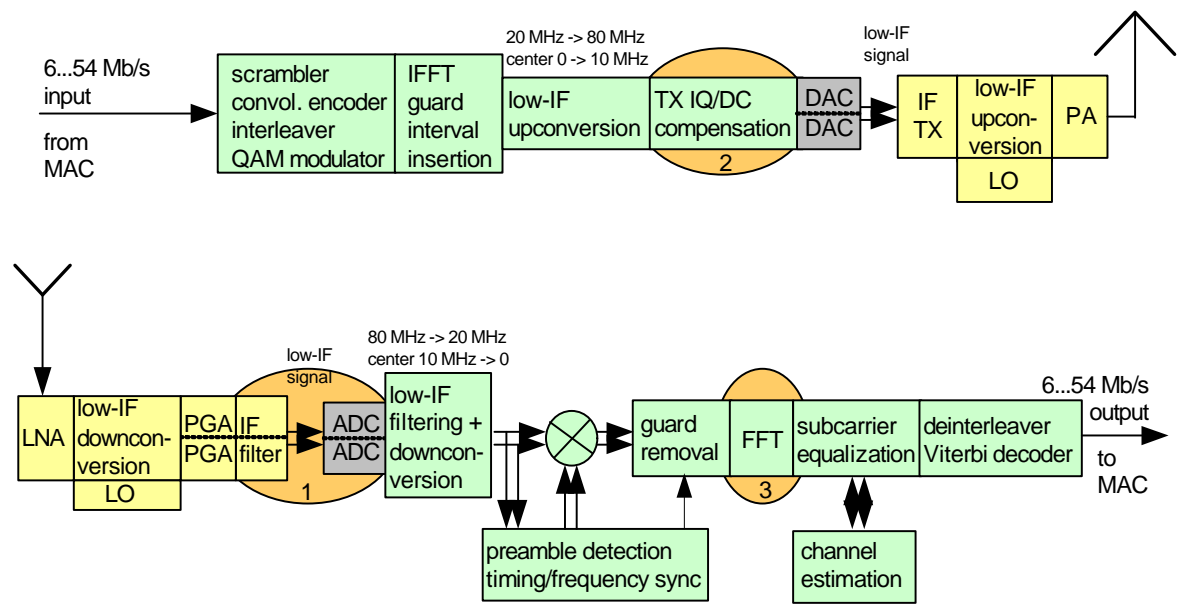

Fig. 7. WLAN OFDM Baseband and RF PHY Transceiver Architecture.

TX/RX antenna switch.

Figure 7 displays the PHY transceiver architecture in more detail. Transmitter baseband processing comprises channel encoding and QAM subcarrier modulation, OFDM modulation (IFFT and guard interval insertion), low-IF upconversion (center of spectrum shifted from 0 to $10 \mathrm{MHz}$ ), TX I/Q and DC compensation, and D/A conversion. Receiver baseband processing performs A/D conversion, channel filtering and low-IF downconversion (center of spectrum shifted back from $10 \mathrm{MHz}$ to 0 ), OFDM demodulation (guard interval removal and FFT), subcarrier equalization, channel decoding, and all necessary ancillary functions such as preamble detection, gain control, timing and frequency synchronization, channel estimation, RF and MAC interfacing, and transceiver control.

In the following, three of the most important aspects of OFDM transceiver implementation are discussed in some detail. Section 3.1 presents the concept of RX channel filtering and A/D conversion (circle 1 in Fig. 7), Sect. 3.2 deals with an example of radio impairment compensation (circle 2), and Sect. 3.3 details on the methodology of designing the FFT unit for OFDM (de)modulation (circle 3).

\subsection{Receiver Channel Filtering and A/D Conversion}

In our channel filtering concept, receiver selectivity is shared between analog IF filtering and digital postfiltering following A/D conversion. The low-IF spectrum mask depicted in the upper part of Fig. 8 reflects the worst-case interference scenario (according to 802.11a) where the desired signal (centered about $10 \mathrm{MHz}$ ) is surrounded by adjacent channel interference (ACI) of some maximum level. The analog polyphase bandpass IF filter (center of Fig. 8) is designed so as to suppress only part of this interference (bottom of Fig. 8); its main purpose is to prevent aliasing and reduce the dynamic range of residual interference in subsequent $\mathrm{A} / \mathrm{D}$ conversion. By virtue of this approach, analog filter complexity and power consumption is much reduced at the expense of only one additional A/D converter bit and the need for a digital postfilter eliminating residual interference.

The filtering concept just described calls for proper choice of the A/D conversion wordlength $W_{A / D}$, which can be derived by means of an $A / D$ conversion level budget such as the one shown in Fig. 9. Starting from the A/D saturation level determined by the particular semiconductor technology (here $600 \mathrm{mVp}$ ), some headroom is added to account 

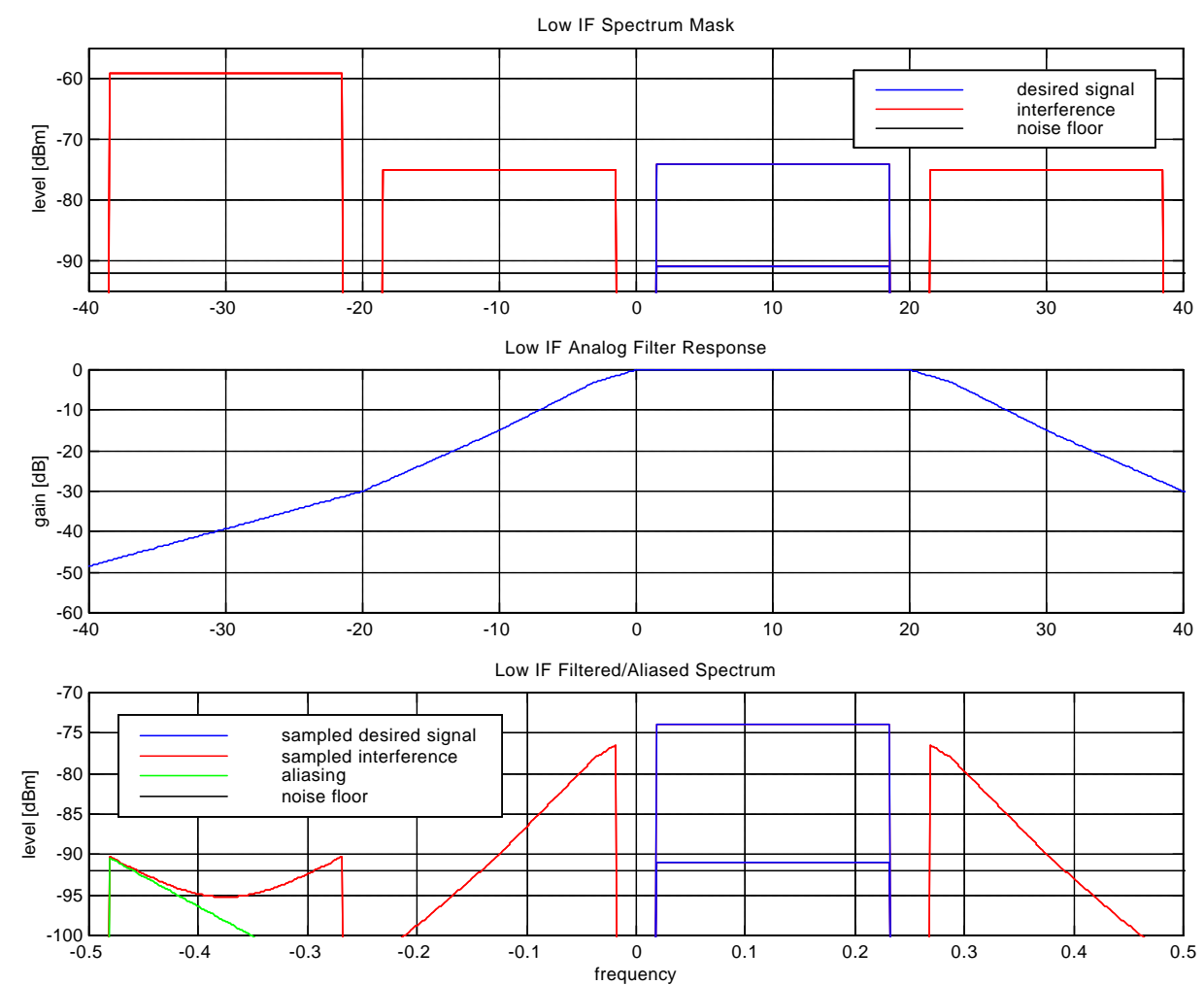

Fig. 8. Low-IF Receiver Channel Filtering and A/D Conversion.

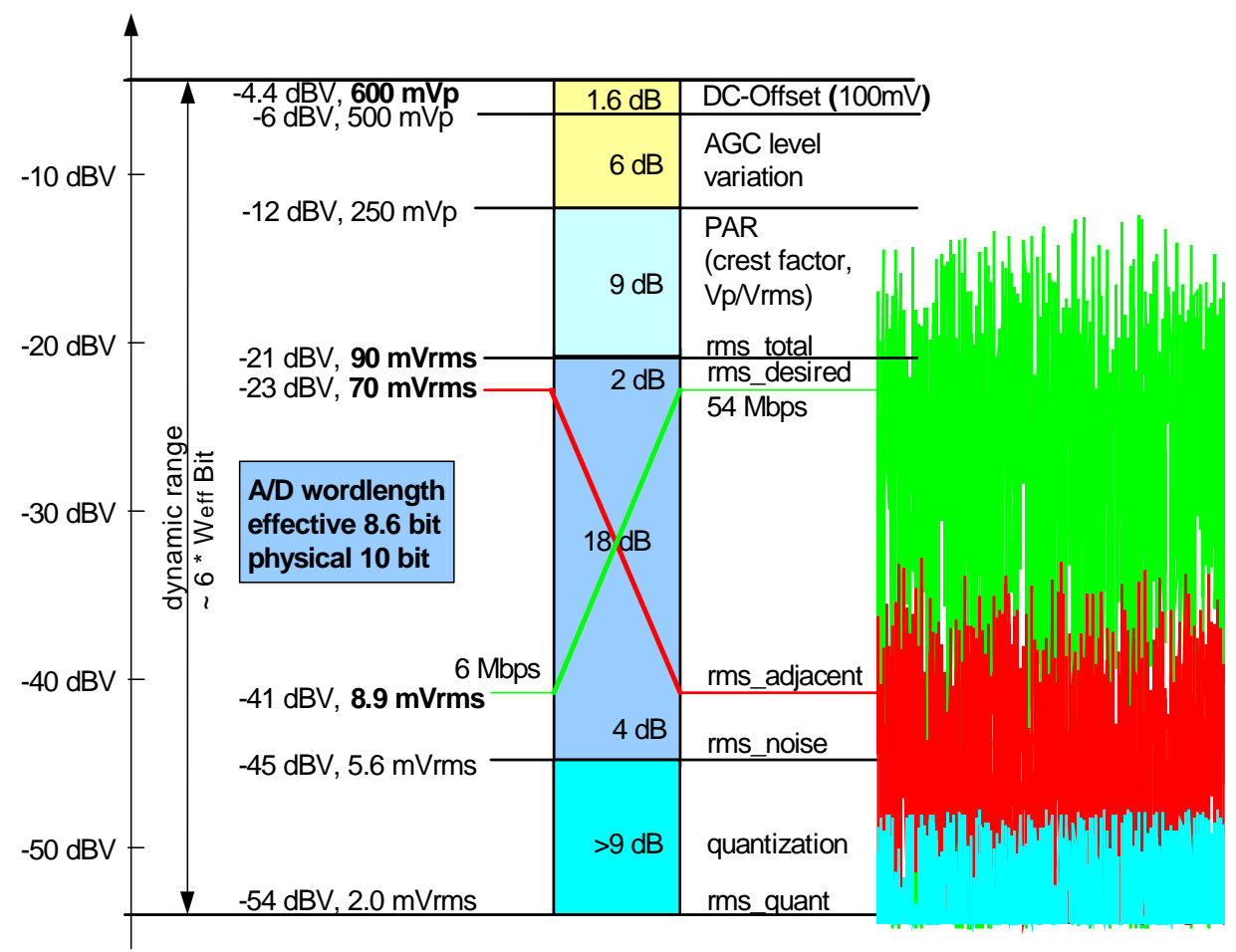

Fig. 9. A/D Conversion Level Budget. 


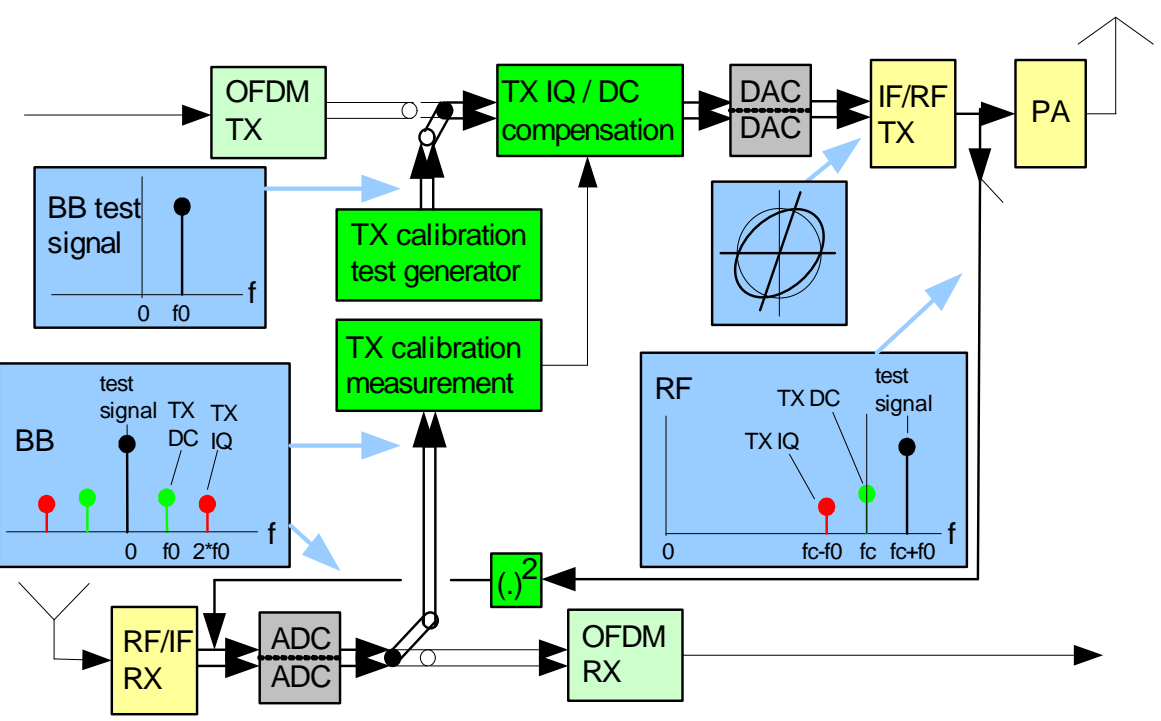

Fig. 10. Calibration Scheme for Transmitter I/Q and DC Compensation.

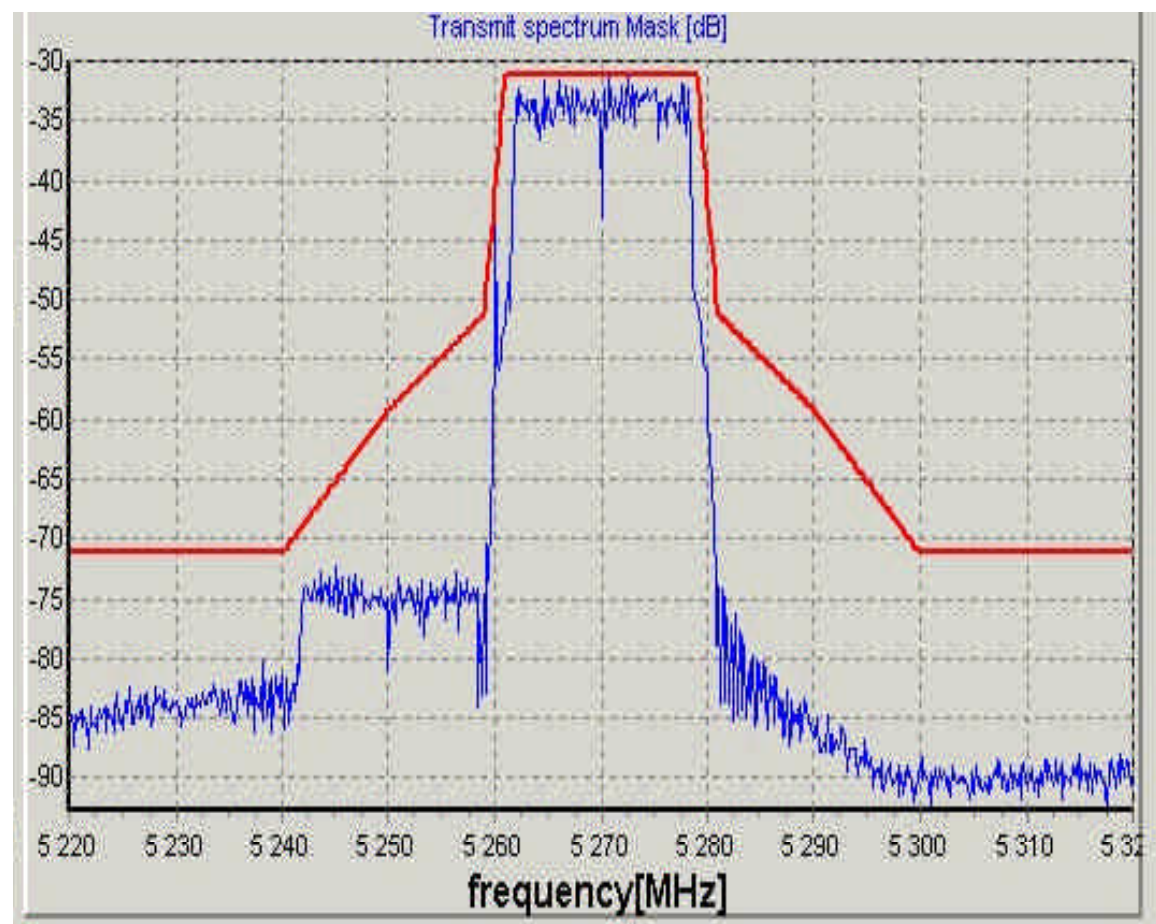

Fig. 11. Performance of Low-IF Transmitter I/Q Compensation.

for various RF/IF receiver imperfections such as DC offset $(1.6 \mathrm{~dB})$ and gain control error $(6 \mathrm{~dB})$. As OFDM signals exhibit a Gaussian-like probability density, their peakto-average ratio (PAR, $9 \mathrm{~dB}$ or more) must be added. The rms level of the composite (desired + residual interference) signal is therefore much smaller than the A/D saturation level $(90 \mathrm{mVrms})$. Depending on the data rate $(6 \ldots .54 \mathrm{Mb} / \mathrm{s})$, the composite signal may comprise more or less amount of interference so that the desired signal level may be further depressed (here to $9 \ldots 70 \mathrm{mVrms}$ ). The required SNR
( $4 \ldots 22 \mathrm{~dB}$ for $6 \ldots 54 \mathrm{Mb} / \mathrm{s})$ then determines the ambient noise level (5.6 mVrms). In order to keep the implementation loss below some predefined limit (here $0.2 \mathrm{~dB}$ ), the A/D quantization level should be lower than the noise level by a considerable margin $(>9 \mathrm{~dB})$. Summing up all contributions, the A/D must cover a dynamic range of almost $50 \mathrm{~dB}$, which corresponds to an effective wordlength of $8.6 \mathrm{~dB}$. Further considering possible nonidealities of the A/D itself, an A/D physical wordlength $W_{A / D}$ of 10 bit has been chosen for implementation. 


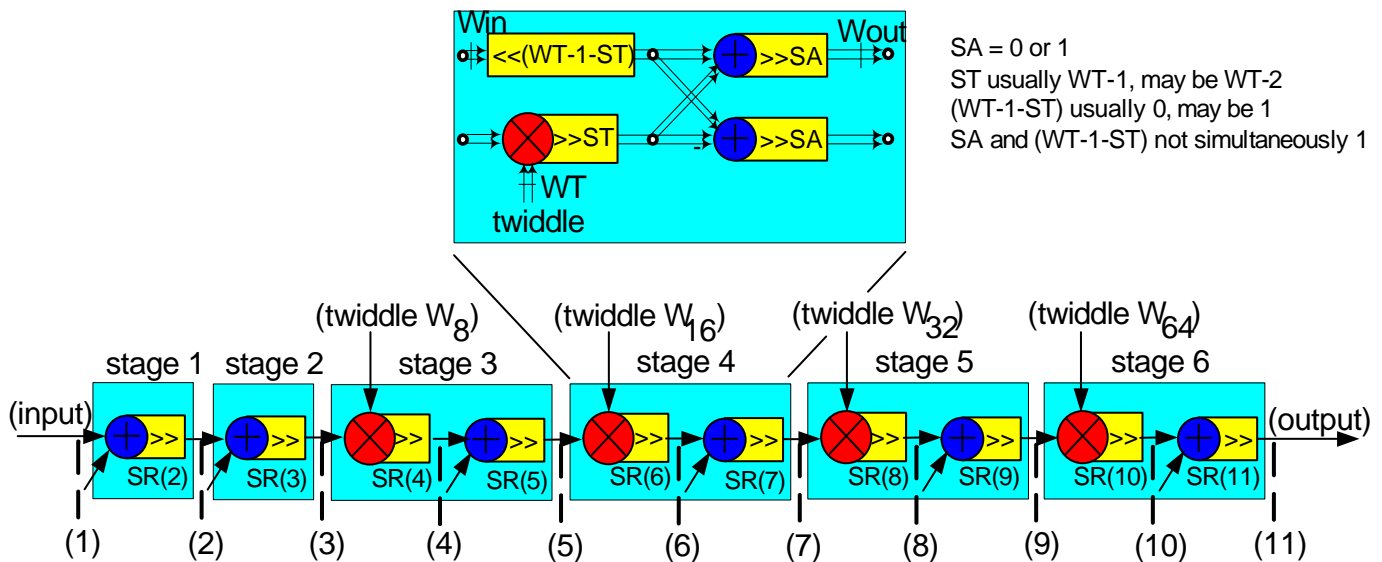

Fig. 12. FFT Architecture.
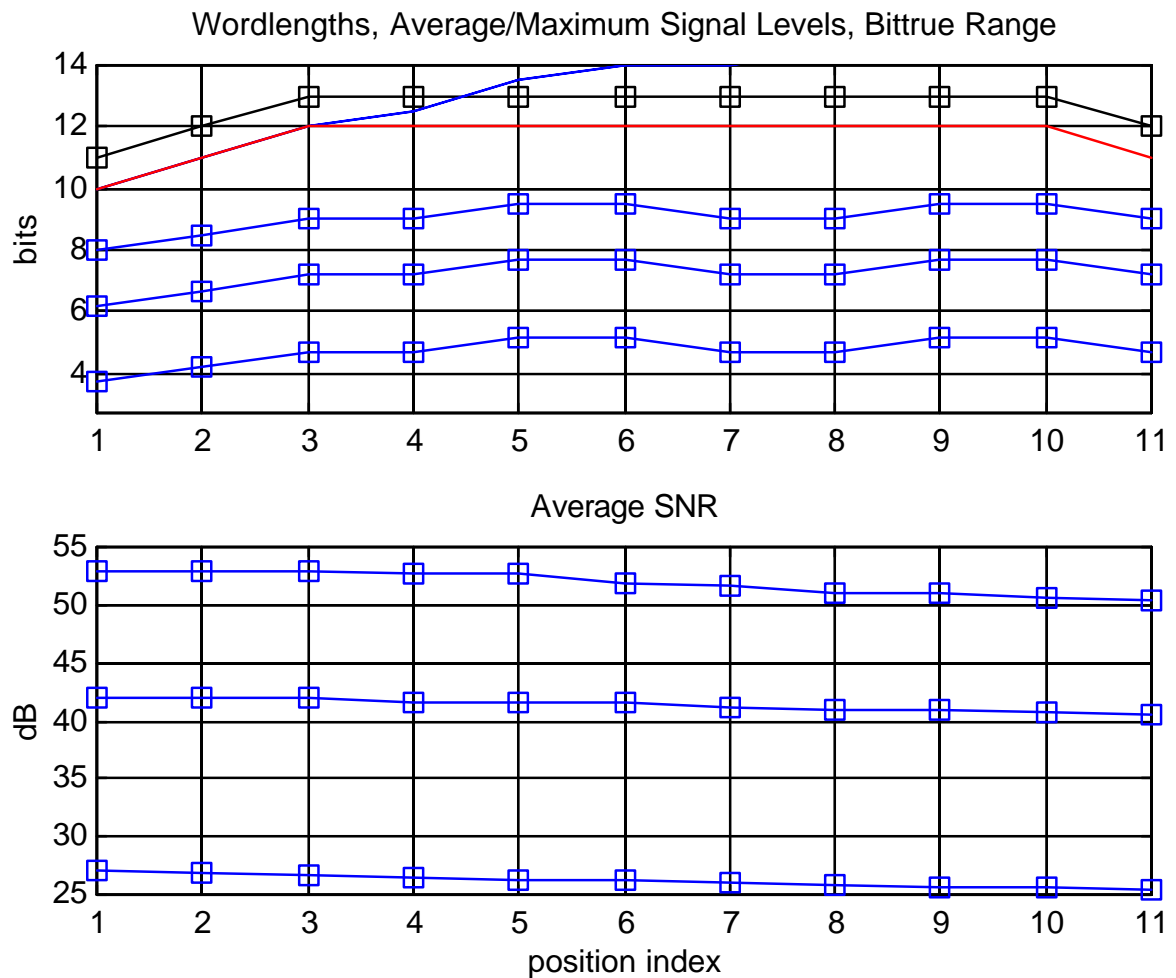

Fig. 13. FFT Wordlength Optimization and Quantization SNR.

3.2 Radio Impairment Compensation: Transmitter I/Q Mismatch

The cost-sensitive mass market calls for direct conversion transceiver architectures. Unfortunately, direct conversion suffers from nonidealities such as DC offsets caused by local oscillator (LO) leakage or I/Q mismatch due to amplitude and/or phase imbalance between the two I (in-phase) and Q (quadrature-phase) rails carrying the complex-valued baseband signal. In the transmitter, such nonidealities lead to the emission of unwanted spurious signals which must be suppressed in order to ensure standard compliance. To this end, the I/Q mismatch and DC calibration scheme shown in Fig. 10 has been implemented.

In calibration mode, a test signal is generated and applied to TX IQ/DC compensation, D/A conversion, and the IF/RF transmitter. The upconverted RF signal is then squared and looped back to the RX A/D converter and from there to the TX calibration measurement unit, see Fig. 10. It can be shown that any I/Q mismatch manifests itself as crosstalk into the opposite sideband, thus compromizing SSB (single sideband) suppression. Upconversion (to carrier frequency 


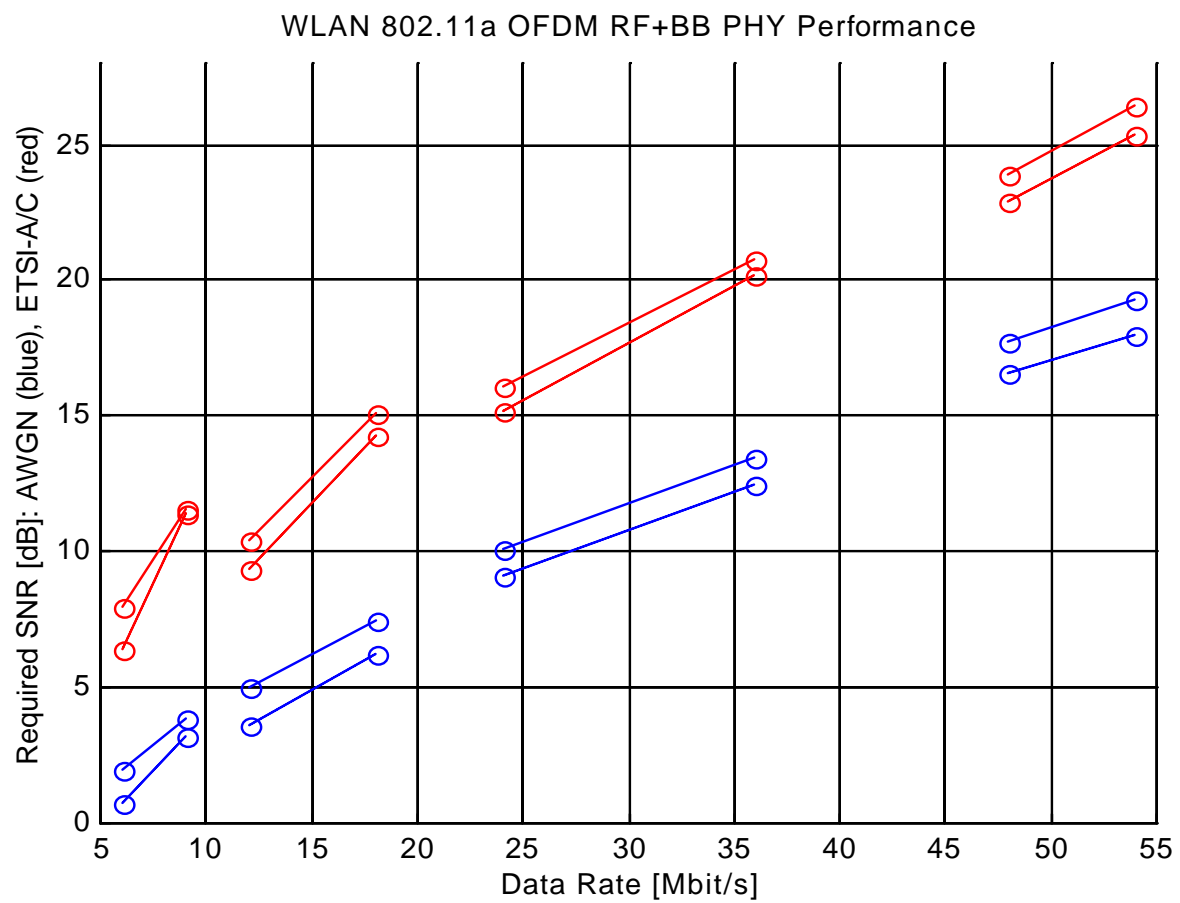

Fig. 14. Performance of Implemented WLAN PHY Transceiver.

$f_{c}$ ) of a complex sinusoidal test signal with (programmable) frequency $+f_{0}$ therefore yields RF signal components at $f_{c}+f_{0}$ (desired), $f_{c}$ (DC offset), and $f_{c}-f_{0}$ (unwanted spur from I/Q mismatch). Squaring the RF signal then yields (real-valued) signal components at $f_{0}$ (DC) and $2 f_{0}$ (I/Q) (left-hand side of Fig. 10) which can be observed by tuning the measurement unit to these frequencies. The IQ/DC compensator settings can now be adapted such that, after several measurement and control cycles, the unwanted signals are suppressed to the extent that their energy is below some predefined threshold. The optimal compensator settings thus found are stored and used during subsequent regular WLAN operation.

I/Q calibration performance thus achieved is illustrated by Fig. 11. The IQ/DC-compensated low-IF transmitter (center frequency $10 \mathrm{MHz}$ upconverted to $5270 \mathrm{MHz}$ ) is seen to improve SSB suppression from $25 . . .30 \mathrm{~dB}$ without compensation to more than $45 \mathrm{~dB}$, thus complying with the $802.11 \mathrm{a}$ TX spectrum mask.

\subsection{FFT Design}

OFDM modulation and demodulation is most efficiently performed by IFFT and FFT processors, respectively. In our implementation, both IFFT and FFT units are based on the same radix-2 pipelined architecture shown in Fig. 12, however with different wordlengths. The 64-point (I)FFT contains six stages, and each stage consists of a butterfly processor element (upper part of Fig. 12) with two input and output ports of wordlength $W_{\text {in }}$ and $W_{\text {out }}$, respectively. Internal butterfly operations comprise twiddle multiplication (complex exponential with wordlength $W_{T}$, complex multiplication, right shift $\left.\gg S_{T}\right)$, a left shift $\ll\left(W_{T}-1-S_{T}\right)$, two complex additions, and two right shifts $\gg S_{A}$ at the output. In the first two stages, twiddle multiplications simplify to additions.

As the 64 input signal samples ripple through the six stages, they are subject to bit-true arithmetic operations which introduce some amount of quantization noise. So from one observation point to the next (1 to 11 in Fig. 12), the quantization noise level continually rises. Focusing on wordlength design for the OFDM demodulator, the art of FFT wordlength design consists in minimizing the wordlengths yet keeping the quantization noise below a certain target level, or, equivalently, keeping the loss in quantization SNR below some limit. As the received and filtered singal may vary in strength, this must hold for a range of input signal levels. In this design, the average FFT input bit-true signal level may deviate considerably from its target value $72(\sim 6$ bit, medium level) and range between about 12 ( $<4$ bit, low AGC gain, strong ACI, $6 \mathrm{Mb} / \mathrm{s}$ mode) and 250 ( $\sim 8$ bit, high AGC gain, no ACI, $54 \mathrm{Mb} / \mathrm{s}$ mode).

In the first step of wordlength optimization, a quantization noise model based on Fig. 12 was developed for fast interactive exploration of the wordlength space. In a second step, the most promising configurations thus identified were verified by simulation. Figure 13 displays the result of wordlength optimization. In the upper drawing, the progression of signal levels across the 11 observation points (Fig. 12) are shown for strong, medium, and weak FFT input signals (three lower curves). From the wordlengths at positions 1 to 11 (upper curve) it is seen that the optimized FFT expands the wordlength by only one bit from 11 bit (input) 
Table 2. WLAN Standard 802.11a/g: PHY Transmission Parameters.

\begin{tabular}{ll}
\hline information data rate & $6,9,12,18,24,36,48,54 \mathrm{Mbit} / \mathrm{s}$ \\
\hline channel bandwidth = sampling rate & $20 \mathrm{MHz}$, occupied bandwidth 16.6 MHz \\
OFDM symbol length & $4.0 \mu \mathrm{s}=80$ samples \\
useful symbol length = FFT length & $3.2 \mu \mathrm{s}=64$ samples \\
guard interval length & $0.8 \mu \mathrm{s}=16$ samples \\
number of occupied subcarriers & 52 \\
number of data subcarriers & 48 \\
number of pilot subcarriers & 4 \\
subcarrier frequency spacing & $312.5 \mathrm{kHz}$ (=20 MHz / 64) \\
subcarrier modulation & BPSK, QPSK,16-QAM, 64-QAM \\
forward error correcting code & convolutional code, K=7, rates 1/2, 2/3, 3/4 \\
\hline
\end{tabular}

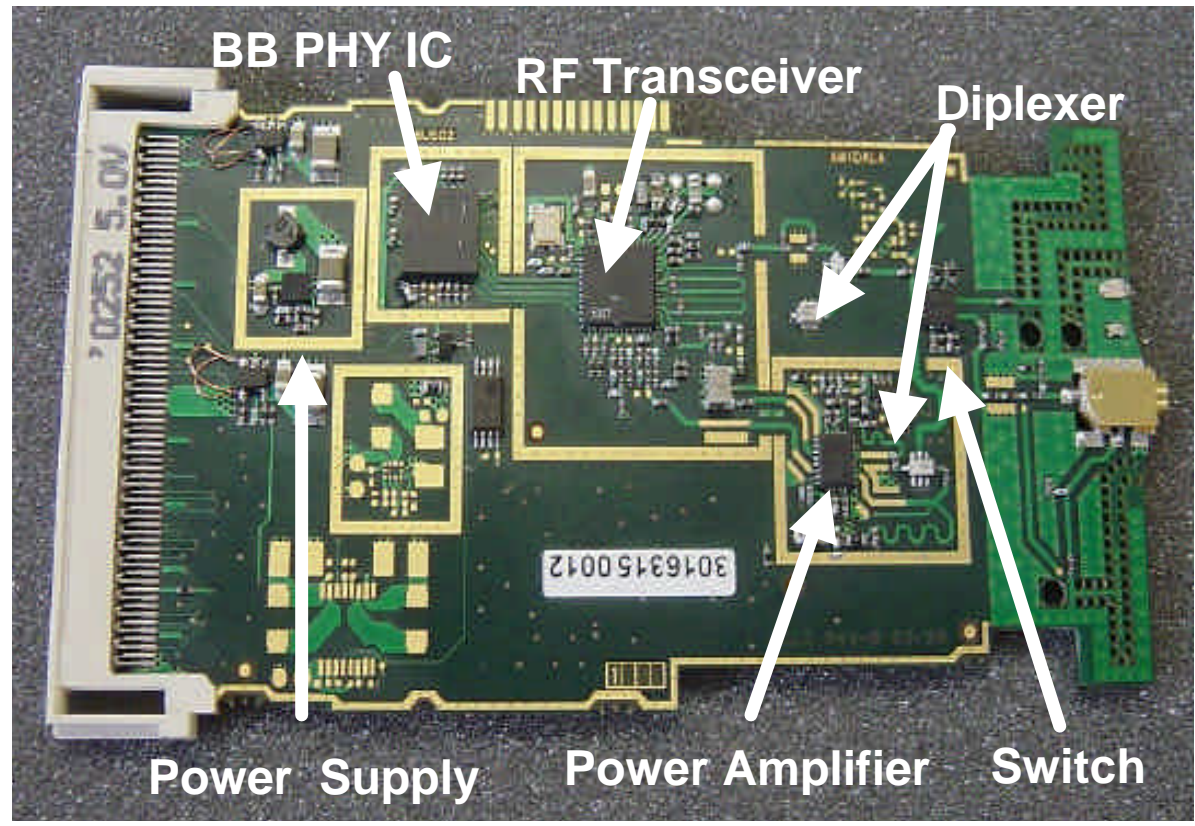

Fig. 15. First-Generation WLAN PHY Transceiver Board.

to 12 bit at its output, which compares favorably with the 6 extra bits (= number of stages) normally required. Also, the bit-true saturation level (= wordlength-1) is more than 2 bits above the maximum average signal level, thus granting more than $12 \mathrm{~dB}$ peak-to-average ratio (PAR) headroom. From the lower drawing of Fig. 13, the loss in quantization SNR across positions 1 to 11 (again for weak, medium, and strong signal levels) is seen to remain small; the quantization noise introduced by the optimized FFT architecture is about $20 \mathrm{~dB}$ below the ambient noise level and therefore negligible at all relevant signal levels.

\section{WLAN Transceiver Performance}

The performance of the implemented WLAN $802.11 \mathrm{a} / \mathrm{g}$ transceiver is shown in Fig. 14 in terms of required SNR versus PHY data rate $6 . . .54 \mathrm{Mb} / \mathrm{s}$. The lower four pairs of curves refer to subcarrier modulation BPSK, QPSK, 16QAM, and 64-QAM, respectively (code rates $1 / 2$ and $2 / 3$ or $3 / 4$, Table 2), and the nonselective AWGN channel, whereas the upper four pairs of curves refer to the same modulation/coding parameters but frequency-selective multipath channels (ETSI channel models $\mathrm{A}$ and $\mathrm{C}$ valid for typical office scenarios). Depending on the data rate, an SNR 
between 8 and $26 \mathrm{~dB}$ is required for these channels. The reference results for ideal floating-point transceiver processing also shown in Fig. 14 (dashed lines) reveal that the implementation loss of the realized radio and baseband PHY device is less than $2 \mathrm{~dB}$, which compares very favorably with other solutions on the market. Figure 15 displays the current WLAN PHY transceiver board housing baseband and radio transceiver chips, power amplifier, and circuitry for antenna switching and power supply. Next-generation implementation are targeting one-chip solutions for the radio and all PHY and MAC processing.

\section{Conclusions}

Despite the presence of multipath, OFDM modulation - featuring a set of smallband subcarriers and a guard interval preserves orthogonality both in time and frequency and thus greatly facilitates channel equalization. Important aspects of OFDM radio and baseband transceiver implementation have been discussed. The concept of sharing receiver channel selection filtering between the analog and digital domains has a number of advantages but requires harmonizing the designs of analog filter, A/D converter, and subsequent digital processing. Radio impairments such as transmitter DC and IQ mismatch are best compensated for by means of digital predistortion with parameters determined by calibration. A methodology for optimizing the performance-complexity tradeoff in OFDM demodulation has been demonstrated on the pipelined FFT architecture and its wordlengths. These and other measures were shown to result in less than $2 \mathrm{~dB}$ implementation loss, which is a very competitive figure of merit for the entire radio and baseband transceiver.

Acknowledgements. The author would like to thank U.R.S.I. and especially K.-J. Langenberg for the invitation to present this paper.

\section{References}

Bingham, J. A. C.: Multicarrier modulation for data transmission: an idea whose time has come, IEEE Commun. Mag., 37, 5-14, May 1990.

Coffey, S., Jones, V. K., Hamady, N., et al.: WWiSE IEEE 802.11n proposal, doc.: IEEE 802.11-04/0935r3, September 2004.

ETSI EN 300 744: Digital Video Broadcasting (DVB); framing structure, channel coding and modulation for digital terrestrial television, V1.4.1, January 2001.

ETSI DVB Document A081: Transmission system for handheld terminals (DVB-H), June 2004.

Fechtel, S. and Blaickner, A.: Efficient FFT and equalizer implementation for OFDM receivers, IEEE Trans. Consumer Electronics, 45, November 1999.
Fechtel, S., Schöllhorn, P., Speth, M., Fock, G., and Schotten, C.: Advanced receiver chip for terrestrial digital video broadcasting: architecture and performance, IEEE Trans. Consumer Electronics, 44, August 1998.

Foschini, G. J.: Layered space-time architecture for wireless communication in a fading environment when using multi-element antennas, Bell Labs Technical Journal, 1, No. 2, 41-59, Autumn 1996.

Foschini, G. J. and Gans, M. J.: On limits of wireless communications in a fading environment when using multiple antennas, Wireless Personal Communications, 6, 311-335, 1998.

Foschini, G. J., Golden, G. D., Valenzuela, R. A., and Wolnianski, P. W.: Simplified processing for high spectral efficiency wireless communication employing multi-element arrays, IEEE Journal on Selected Areas of Communications, 17, No. 11, 1841-1852, November 1999.

IEEE 802.11a: Wireless LAN medium access control (MAC) and physical layer (PHY) specifications: high speed physical layer in the $5 \mathrm{GHz}$ band, July 1999 .

IEEE 802.11b: Standard for wireless LAN medium access control (MAC) and physical layer (PHY) specifications, 2001.

IEEE 802.11g: Wireless LAN medium access control (MAC) and physical layer (PHY) specifications further higher-speed physical layer extension in the $2.4 \mathrm{GHz}$ band, Supplement to IEEE Standard, 2003.

Jones, V. K., Raleigh, G., and van Nee, R.: MIMO answers highrate WLAN call, EE Times, December 2003.

Kammeyer, K. D., Schmidt, H., Rückriem, R., and Fechtel, S.: OFDM: An old idea solves new problems, in: Proc. Int. Symp. Theoretical Electrical Engineering (ISTET), 2, Linz, Austria, August 2001.

Meyr, H., Moeneclaey, M., and Fechtel, S.: Digital communication receivers, Wiley Interscience, 1997.

Mujtaba, A., Stephens, A. P., Purkovic, A., et al.: TGn Sync complete proposal, IEEE presentation, September 2004.

Pawlowski, S.: Motivation for a reconfigurable wireless architecture, Intel Labs, January 2003.

Pirsch, P.: Architectures for digital signal processing, Wiley Interscience, 1998.

Speth, M., Fechtel, S., Fock, G., and Meyr, H.: Optimum receiver design for wireless broadband systems using OFDM - Part I, IEEE Trans. Commun., 47, November 1999.

Speth, M., Fechtel, S., Fock, G., and Meyr, H.: Optimum receiver design for OFDM-based broadband transmission - Part II: A case study, IEEE Trans. Commun., 49, April 2001.

Vermeer, V. and Cramer, M.: Wireless eyes throughput challenge, EE Times, December 2003.

Wireless Gigabit with advanced multimedia support (WIGWAM), Project Proposal, June 2003.

Wolnianski, P. W., Foschini, G. J., Golden, G. D., and Valenzuela, R. A.: V-BLAST: an architecture for realizing very high data rates over the rich-scattering wireless channel, 1998 URSI International Symposium on Signals, Systems, and Electronics, 295300, October 1998. 